\title{
Comparison of asynchronous versus synchronous arm crank ergometry
}

\author{
K Mossberg*,1, C Willman ${ }^{1}$, MA Topor ${ }^{1}, \mathrm{H} \mathrm{Crook}^{1}$ and S Patak ${ }^{1}$ \\ ${ }^{1}$ Department of Physical Therapy, University of Texas Medical Branch, Galveston, Texas, USA
}

\begin{abstract}
Study Design: A direct comparison of synchronous versus asynchronous arm crank ergometry has not been carried out previously. Therefore, a comparative research design was employed.

Objective: To assess the physiological responses of arm cranking when performed asynchronously (arms moving opposite to each other) versus synchronously (both arms moving in the same direction simultaneously).

Setting: A university hospital setting in Galveston, Texas, USA.

Methods: Seventeen individuals between the ages of 19 and 53 years were studied, 11 with paraplegia and six with no apparent disability. Two maximal arm crank graded exercise tests were performed with the subject seated in a wheelchair. Testing consisted of both arms (1) asynchronously (reciprocally) pushing and pulling the crank handles and (2) pushing and pulling the crank handles synchronously. Each test consisted of 2 min stages starting at $20 \mathrm{~W}$ and increasing $10 \mathrm{~W}$ per stage thereafter until exhaustion. Heart rate, oxygen consumption, and minute ventilation were measured and recorded during each stage. Blood lactate levels were monitored before and after each test. Statistical analysis was performed using the multivariate Hotelling's $\mathrm{T}^{2}$ followed by post hoc univariate tests.

Results: Greater power and longer test times (both groups, $P<0.05$ ) and higher post test blood lactates (nondisabled $P<0.01$, paraplegic $P<0.05$ ) were achieved with asynchronous cranking versus synchronous cranking. While submaximal responses were similar between the two modes of cranking, there was a tendency for all variables to be lower with asynchronous. All subjects preferred asynchronous rather than synchronous cranking.

Conclusion: Despite few statistically significant differences, based on the subjective reports from all subjects, we believe there is a clinically significant difference between the two modes of cranking. The results suggest that the mode of cranking may have implications for arm crank testing, training, and functional locomotion in individuals with lower extremity impairments.
\end{abstract}

Keywords: spinal cord injury; exercise testing; blood lactate; heart rate; oxygen consumption; minute ventilation

\section{Introduction}

Arm ergometry and upper extremity training methods for cardiorespiratory fitness are of particular concern to individuals who rely on their upper extremities for wheelchair locomotion. Assessment of and training for upper body aerobic fitness has taken many forms but almost exclusively consists of asynchronous, (ie, reciprocal) arm cranking. ${ }^{1-4}$ However, the use of standard wheelchairs requires that propulsive force be applied to the handrims in a synchronous manner, rather than asynchronously.

The use of asynchronous or reciprocal movement patterns during arm ergometry studies is most likely

*Correspondence: KA Mossberg, Department of Physical Therapy, School of Allied Health Sciences, University of Texas Medical Branch, 301 University Boulevard, Galveston, TX 77555-1028, USA the result of the fact that arm crank ergometers evolved from modified leg cycle ergometers. Bobbert ${ }^{5}$ was the first to describe synchronous arm cranking and later Shaw and colleagues ${ }^{6}$ reported on synchronous arm cranking as a mode of evaluating patients with ischemic heart disease. Very little work has been reported comparing the effects of asynchronous and synchronous arm cranking, particularly in individuals with lower extremity impairments. More attention to individuals with paraplegia would seem warranted because of deficiencies in muscle innervation and trunk stability. We are aware of just two reports that have made direct comparisons between synchronous and asynchronous modes of arm cranking. ${ }^{7,8}$ Only three individuals with paraplegia were studied and the report lacks sufficient detail necessary to draw conclusions. $^{7}$ Both studies recommend further study of individuals with spinal impairments. 
Anecdotal evidence from persons with spinal cord injury (SCI) suggests that asynchronous arm cranking is easier to perform and more efficient than synchronous cranking. Furthermore, in studies on wheelchair propulsion, applying force to the push rims asynchronously has been shown to be more efficient than synchronous application of force. ${ }^{9,10}$ In addition, alternate modes of arm powered locomotion exist and are being developed to improve the efficiency of movement for the disabled. Given these factors and the paucity of comparative data, the purpose of this study was to compare the physiological responses of individuals with and without disability when performing synchronous versus asynchronous arm crank exercise. We hypothesized that asynchronous cranking would elicit higher peak and lower submaximal responses for key physiologic variables such as heart rate, oxygen consumption and pulmonary ventilation.

\section{Methods}

\section{Subjects}

Six nondisabled males and 11 individuals with paraplegia between the ages of 19 and 53 years (Table 1) gave informed written consent to participate. The Institutional Review Board of the University of Texas Medical Branch at Galveston approved all procedures. All subjects with paraplegia were male except for one female (Table 2). Subjects who exhibited apparent cardiopulmonary disease risk factors or upper extremity neuromusculoskeletal impairments were excluded from the study.

\section{Instrumentation}

A metabolic cart (Gould 9000 Cardiopulmonary Exercise System, Gould Inc., Cardiopulmonary Products Div., Dayton, OH, USA) was used to determine oxygen consumption (paramagnetic $\mathrm{O}_{2}$ analyzer), carbon dioxide production (infra-red $\mathrm{CO}_{2}$ analyzer), and volumes (dry rolling seal spirometer) from expired breath samples taken at rest and during each test. Calibration took place immediately prior to and after each test using known concentrations of oxygen and carbon dioxide. An electronically braked arm ergometer (Tru-Kinetics UpperCycle, Henly International Inc., Sugarland, TX, USA) was utilized for performance of the graded exercise tests. Orientation of the crank handles was easily changed to accommodate either asynchronous or synchronous motion. The

Table 1 Subject characteristics

\begin{tabular}{lrcc}
\hline & $\mathrm{n}$ & Age (years) & Weight $(\mathrm{kg})$ \\
\hline Nondisabled & 6 & $39.2 \pm 2.2$ & $85.4 \pm 2.4$ \\
Paraplegic & 11 & $34.9 \pm 3.1$ & $72.6 \pm 3.6$ \\
\hline
\end{tabular}

Values are mean $\pm \mathrm{SE}$
Table 2 Characteristics of the disabled subjects

\begin{tabular}{lccccc}
\hline Subject & Age & Gender & Level & Duration of impairment \\
\hline 1 & 27 & $\mathrm{~m}$ & T5 & 7 years & 11 months \\
2 & 39 & $\mathrm{~m}$ & T6 & 5 years & 3 months \\
3 & 45 & $\mathrm{~m}$ & T8 & 29 years & 8 months \\
4 & 36 & $\mathrm{~m}$ & T9 & 3 years & 2 months \\
5 & 22 & $\mathrm{f}$ & T9 & 2 years & 3 months \\
6 & 44 & $\mathrm{~m}$ & T11 & 1 year & 1 month \\
7 & 44 & $\mathrm{~m}$ & T12 & 15 years & 7 months \\
8 & 23 & $\mathrm{~m}$ & T12 & 2 years & 3 months \\
9 & 40 & $\mathrm{~m}$ & L1 & 18 years 1 month \\
10 & 45 & $\mathrm{~m}$ & L2 & 1 year & 3 months \\
11 & 19 & $\mathrm{~m}$ & L5 & 18 years & 10 months \\
\hline
\end{tabular}

radius of rotation was either 12.5 or $19 \mathrm{~cm}$ depending on the arm length of the person being tested. Blood lactate levels were determined from approximately 15 microliters of finger prick blood. An automated glucose/lactacte analyzer performed the analysis (2300 Stat Glucose/L-Lactate Analyzer, Yellow Springs Instruments Co., Yellow Springs, OH, USA). The electrocardiogram and heart rate were continuously monitored with a single lead (CM5 configuration) ECG (MDE Escort ECG Monitor Medical Data Electronics, Arleta, CA, USA). Heart rate was also continuously monitored by a portable heart rate monitoring system (Polar Vantage XL Heart Rate Monitor Polar CIC Incorporated, Port Washington, NY, USA) and stored data was later downloaded to a personal computer.

\section{Procedure}

Subjects completed two graded arm crank tests in random order, one synchronous and the other asynchronous. Exercise tests were administered approximately 5-10 days apart. The protocols for each test were identical in terms of work and time within each stage.

Nondisabled subjects were seated in a standard wheelchair with their feet placed on the footrests. Placement of their feet on the footrests reduced their ability to use the lower extremities for stabilization. In addition, nondisabled subjects were verbally discouraged from doing so. Individuals with paraplegia performed the tests in their everyday wheelchairs. For all subjects, the wheelchair was positioned at a comfortable distance from the ergometer allowing for a slight bend $\left(15-20^{\circ}\right)$ of the subject's elbow during maximal reach. The axis of rotation of the arm crank was horizontally level with the axis of rotation of the shoulder. Adjustable straps and cables stabilized the wheelchair and ergometer to a floor supported platform.

During each graded exercise test the initial workload was $20 \mathrm{~W}$ and was increased $10 \mathrm{~W}$ every $2 \mathrm{~min}$ thereafter. Subjects were instructed to maintain a cranking rate between 50 and 60 revolutions per minute (rpm). Testing was terminated when the subject 
was unable to maintain the required cranking rate or upon the subject's volitional fatigue. Subjects were given a $1 \mathrm{~min}$ rest period every $6 \mathrm{~min}$ at which time blood pressure was measured. Heart rate, $\mathrm{VO}_{2}, \mathrm{~V}_{\mathrm{E}}$, and ECG were monitored throughout each test. Rating of perceived exertion (RPE) was determined at the completion of each 2 min stage using a modified Borg scale. 11

\section{Data Analysis}

All descriptive data are presented as means and standard errors. A comparative design was used in this study in which $\mathrm{HR}, \mathrm{V}_{\mathrm{E}}, \mathrm{VO}_{2}$, and blood lactate responses were examined. The independent variable in this study was the type of test performed, asynchronous versus synchronous. Statistical comparisons were made using the multivariate Hotelling's $\mathrm{T}^{2} \cdot{ }^{12}$ The alpha level was set at 0.05 . In the event that a level of significance was reached, post hoc univariate tests were run to determine which dependent variable was different between asynchronous and synchronous cranking modes. All data were analyzed using statistical software for a personal computer (Systat 6.0, SPSS Inc., Chicago, IL, USA).

\section{Results}

The mean peak responses attained during asynchronous and synchronous arm cranking are presented in Table 3. Overall, peak responses for both groups of individuals were higher when cranking asynchronously versus synchronously. However, only total test time and posttest blood lactate levels were statistically higher during asynchronous cranking. The longest total test time of 29.6 min achieved by the nondisabled cranking in the asynchronous mode was equivalent to generating $140 \mathrm{~W}$ of power for $1.6 \mathrm{~min}$. The shortest total test time of $22.6 \mathrm{~min}$ achieved by the paraplegics cranking in the synchronous mode was equivalent to a power output of $110 \mathrm{~W}$ for $1.6 \mathrm{~min}$. All individuals were able to either generate greater power or maintain a given power output for a longer period of time cranking in the asynchronous mode.

The peak heart rate responses for the two modes of cranking were similar within the two groups (Table 3 ). When examining the submaximal heart rate responses (Figure 1) there was no difference between modes of cranking although slightly higher heart rates were seen during synchronous cranking during most minutes of the exercise.

Table 3 Peak responses for asynchronous and synchronous arm cranking performed by nondisabled $(n=6)$ and paraplegic $(n=11)$ subjects

\begin{tabular}{lccc}
\hline Variable & Asynchronous & Synchronous \\
\hline Total test time (min) & & & \\
$\quad$ Nondisabled & $29.6 \pm 2.5$ & $*$ & $25.2 \pm 1.9$ \\
$\quad$ Paraplegic & $25.2 \pm 2.1$ & $*$ & $22.6 \pm 1.9$ \\
Heart rate (b.p.m.) & $163 \pm 10$ & & $158 \pm 8$ \\
$\quad$ Nondisabled & $180 \pm 5$ & & $176 \pm 4$ \\
$\quad$ Paraplegic & $1704 \pm 105$ & & $1550 \pm 99$ \\
Oxygen uptake (mL/min) & & $1348 \pm 78$ \\
$\quad$ Nondisabled & $1405 \pm 102$ & & \\
$\quad$ Paraplegic & & & $65.1 \pm 5.3$ \\
Minute ventilation (L/min) & & & \\
$\quad$ Nondisabled & $71.7 \pm 8.2$ & & $59.7 \pm 5.3$ \\
$\quad$ Paraplegic & $64.2 \pm 6.8$ & & \\
Lactate (mL/dL) & $8.34 \pm 0.67$ & $* *$ & $6.45 \pm 0.49$ \\
$\quad$ Nondisabled & $8.51 \pm 0.48$ & $*$ & $7.30 \pm 0.37$ \\
$\quad$ Paraplegic &
\end{tabular}

Values are mean $\pm \mathrm{SE} ; * P<0.05 ; * * P<0.01$
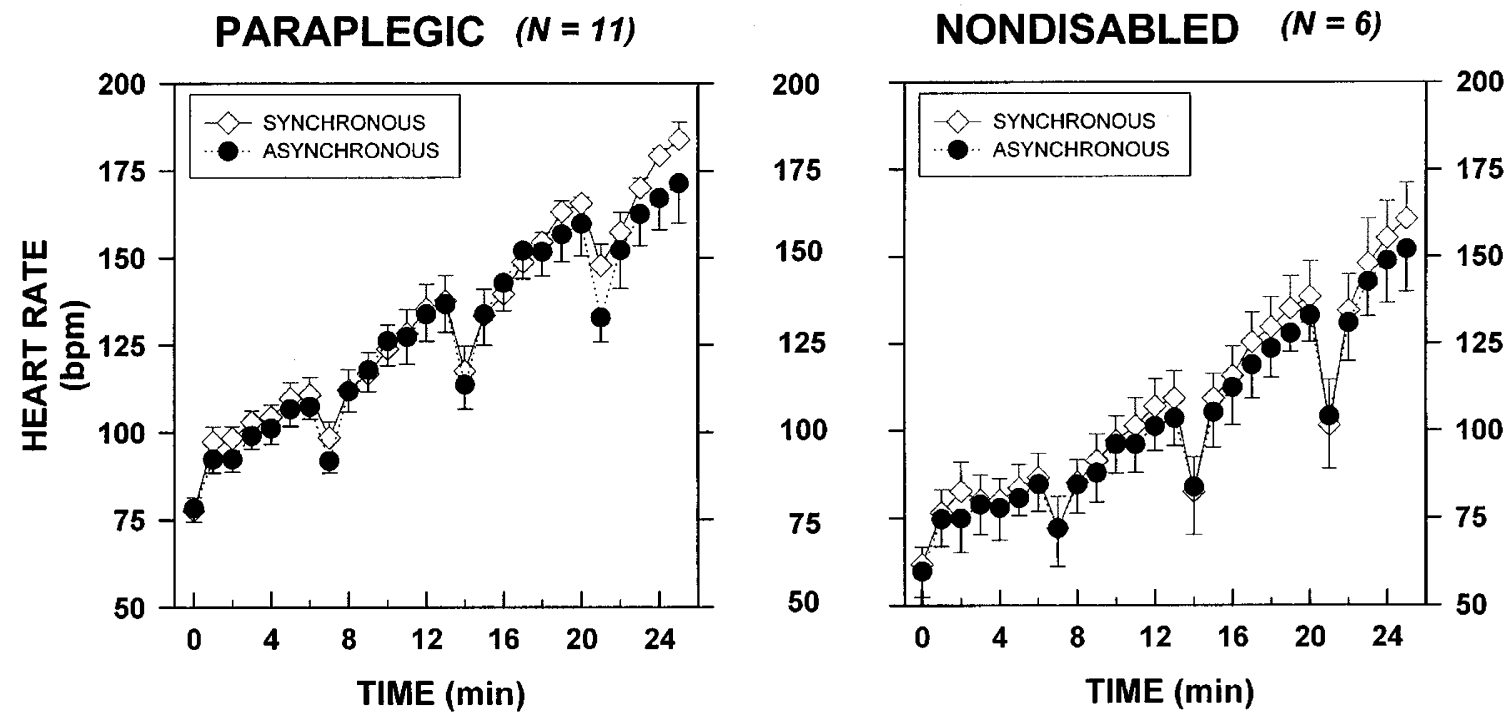

Figure 1 Submaximal heart rate responses during asynchronous and synchronous arm cranking. Values are mean \pm SE 
The peak oxygen consumption values for the two modes of cranking were not significantly different when comparing asynchronous and synchronous cranking. However, there was a strong tendency for asynchronous cranking to elicit higher oxygen uptakes, especially in the nondisabled. This is consistent with the higher power and total test times achieved when cranking asynchronously. Comparisons of submaximal oxygen consumption revealed no differences during the two test modes (Figure 2). However, synchronous cranking required slightly higher submaximal $\mathrm{VO}_{2}$ during most minutes of exercise for both groups.

Minute ventilation also was found to be similar statistically between modes of cranking. However, like
$\mathrm{HR}$ and $\mathrm{VO}_{2}$, was a tendency for the synchronous mode to elicit lower peak pulmonary ventilation rates. Submaximal pulmonary ventilation (Figure 3) tended to be higher during synchronous cranking in both groups but not significantly.

\section{Discussion}

Individuals with spinal cord injury are limited in the type of exercises they can participate in secondary to their inability to voluntarily use their lower extremities. We studied submaximal and peak responses to one of the most common forms of upper body exercise available to the disabled population, asynchronous
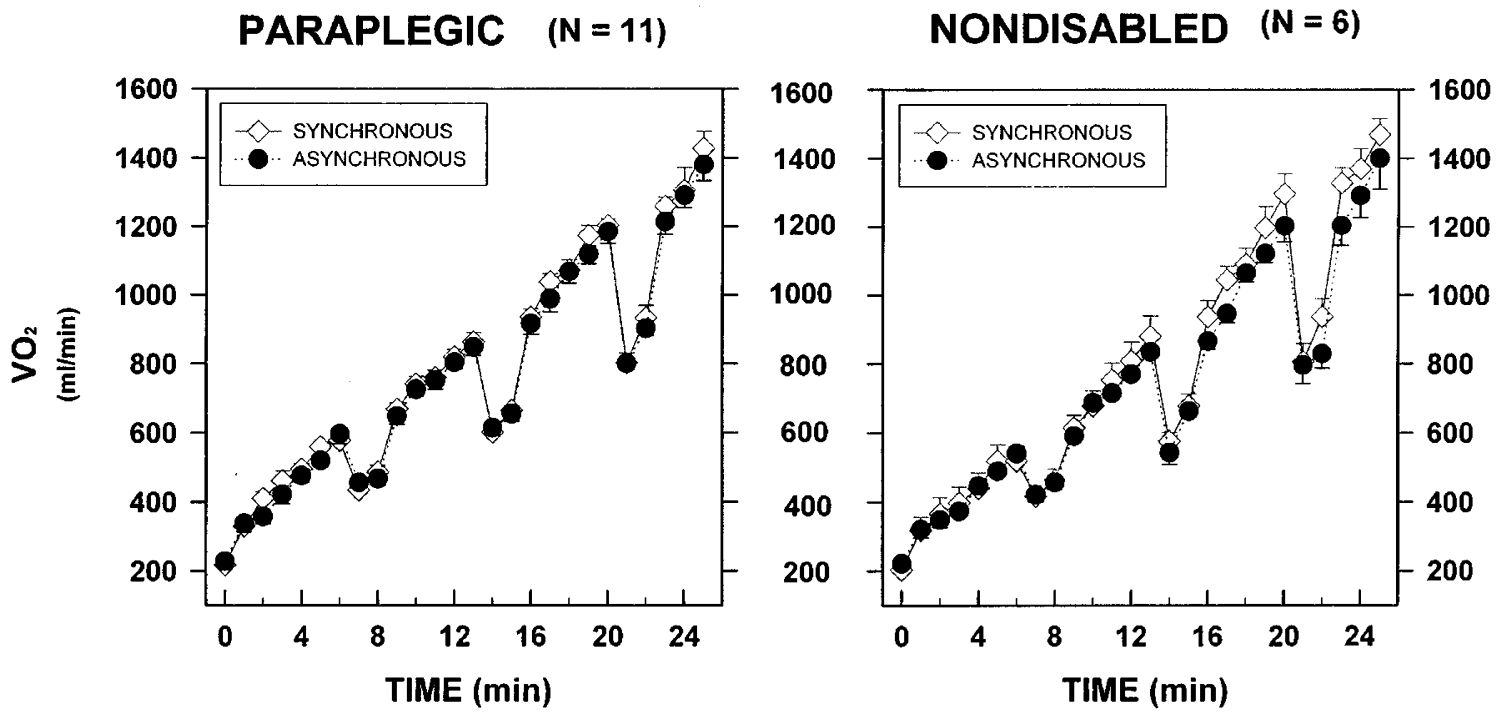

Figure 2 Submaximal $\mathrm{VO}_{2}$ responses during asynchronous and synchronous arm cranking. Values are mean $\pm \mathrm{SE}$
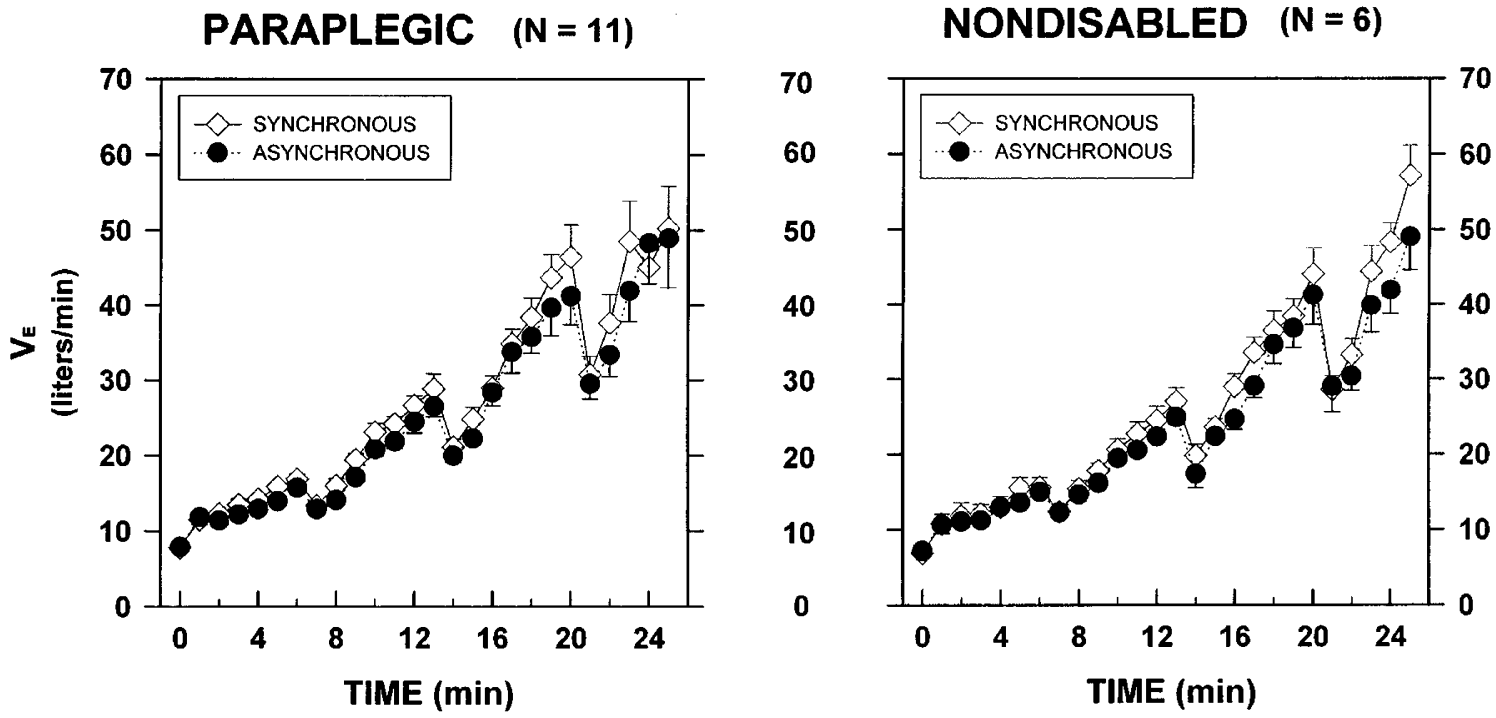

Figure 3 Submaximal $V_{E}$ responses during asynchronous and synchronous arm cranking. Values are mean $\pm \mathrm{SE}$ 
arm cranking, and compared it to the less common synchronous mode of arm cranking. We found that all subjects were able to generate greater power and higher blood lactates at peak exercise with asynchronous cranking, but only slight differences existed during submaximal levels of work. Subjectively, all participants preferred the asynchronous mode of cranking to the synchronous mode.

Little has been reported with respect to the submaximal responses to asynchronous and synchronous cranking, especially in individuals with paraplegia. Marincek and colleagues ${ }^{7}$ studied three individuals with paraplegia at power outputs of 25 , 50 and $75 \mathrm{~W}$. Unfortunately, their report is unclear regarding the specific responses to these two modes of exercise, making it impossible to determine whether there were differences between asynchronous and synchronous modes of arm cranking in either the three individuals with paraplegia or the five without disability.

Hopman and coworkers ${ }^{8}$ studied ten nondisabled individuals and reported no differences in peak responses between the two modes of cranking. Measures of efficiency during submaximal work were found to be no different either. However, as with all our subjects, the majority of their subjects preferred asynchronous to synchronous cranking.

It is well known that peak performance during upper extremity ergometry is limited by local muscle fatigue rather than central cardiorespiratory mechanisms. ${ }^{2,13}$ In our subjects cessation of exercise was almost exclusively the result of triceps brachii fatigue. This occurred sooner during the synchronous form of cranking with the anterior deltoid muscles also contributing significantly to the performance limitation. The more pronounced triceps fatigue during synchronous cranking is consistent with previous findings that showed triceps to be activated during $75 \%$ of the synchronous cranking cycle versus $50 \%$ during the asynchronous movement cycle. ${ }^{8}$ During asynchronous cranking the forward 'push' developed by the triceps and the shoulder flexors is assisted by the backward 'pull' of the contralateral elbow flexors and shoulder extensors. As a result, fatigue of the triceps was delayed and probably accounted for the greater total test times and longer power production during asynchronous cranking.

We believe the results of our study may have implications for training of the upper extremities of those who use wheelchairs. Rodgers and colleagues ${ }^{14}$ found that the force applied to the handrim of the wheelchair was strongly correlated with the isokinetic torques of the anterior deltoid and triceps muscle groups. In addition, during fatiguing wheelchair propulsion, activation of the anterior deltoid increased as a function of percent cycle time. Their results, along with common rehabilitation practices, suggest that specific overload of the triceps and anterior deltoid muscles would improve functional independence of the wheelchair user. The subjective reports of our subjects and EMG data ${ }^{8}$ suggest that synchronous arm cranking may be a simple and convenient method to perform overload training of the elbow extensors and shoulder flexors.

Furthermore, the synchronous mode of arm cranking is consistent with synchronous wheelchair propulsion. The functional requirement of simultaneously flexing and extending right and left shoulders and arms suggests that a similar movement pattern be employed when a wheelchair user trains the upper extremities on an arm ergometer. Several investigations have demonstrated the principle of exercise specificity in very general terms, ${ }^{15,16}$ for lower extremity training adaptations, ${ }^{17,18}$ and transfer of training between the arms and legs. ${ }^{19}$ The specificity of training adaptations would suggest that further research is necessary to compare the relative merits of training asynchronously versus synchronously to test the principle of exercise training specificity in individuals with tetra- and paraplegia.

In conclusion, compared to asynchronous arm cranking, synchronous arm cranking brought about earlier arm fatigue, lower power outputs and slightly lower peak physiological responses. Consequently, asynchronous cranking may be a better form of testing peak performance in both individuals with spinal cord injury and those whom otherwise cannot perform a lower extremity progressive exercise test. However, for the wheelchair user, synchronous cranking is more task specific and perhaps aerobic conditioning programs should be designed that include synchronous movement patterns of the upper extremities similar to wheelchair propulsion, especially early in the rehabilitation process.

\section{Acknowledgements}

This project was funded in part by a grant from the Texas Physical Therapy Education and Research Foundation.

\section{References}

1 Williams J, Cottrell E, Powers SK, McKnight T. Arm ergometry: a review of published protocols and the introduction of a new weight adjusted protocol. J Sports Med 1983; 23: 107-112.

2 Franklin BA. Exercise testing, training and arm ergometry. Sports Med 1985; 2: 100-119.

3 Celli BR. The clinical use of upper extremity exercise. Clin Chest Med 1994; 15: 339-349.

4 Franklin BA. Diagnostic and functional exercise testing: test selection and interpretation. J Cardiovasc Nurs 1995; 10: 8-29.

5 Bobbert AC. Physiological comparison of three types of ergometry. J Appl Physiol 1960; 15: 1007 - 1014.

6 Shaw DJ et al. Arm-crank ergometry: a new method for the evaluation of coronary artery disease. Am J Cardiol 1974; 33: $801-805$.

7 Marincek CRT, Valencic V. Arm cycloergometry and kinetics of oxygen consumption in paraplegics. Paraplegia 1977-78; 15: $178-185$

8 Hopman MTE et al. Physiological responses to asynchronous and synchronous arm-cranking exercise. Eur J Appl Physiol 1995; 72: $111-114$ 
9 Engel P, Hildebrandt G. Wheelchair design - Technological and physiological aspects. Proc Roc Soc Med 1974; 67: 409-413.

10 Glaser RM, Sawka MN, Young RE, Suryaprasad AG. Applied physiology for wheelchair design. J Appl Physiol 1980; 48: $41-$ 44.

11 Borg GAV. Psychophysical bases of perceived exertion. Med Sci Sports Exerc 1982; 14: 377-381.

12 Portney LG, Watkins MP. Foundations of Clinical Research: Applications to Practice. Norwalk, CT: Appleton and Lange; 1993.

13 Sawka MN. Physiology of upper-body exercise. In Exercise and Sport Sciences Reviews Vol. 14, Macmillan Publishing Co. New York 1986: pp 175-211.

14 Rodgers MM et al. Biomechanics of wheelchair propulsion during fatigue. Arch Phys Med Rehabil 1994; 75: 85-93.
15 DeLorme TL. Restoration of muscle power by heavy-resistance exercises. J Bone Jt Surg 1945; 27: 645-667.

16 Dudley GA, Djamil R. Incompatibility of endurance- and strength-training modes of exercise. J Appl Physil 1985; 59: $1446-1451$.

17 Pechar GS et al. Specificity of cardiorespiratory adaptation to bicycle and treadmill training. $J$ Appl Physiol 1974; 36: 753-756.

18 Roberts JA, Alspaugh JW. Specificity of training effects resulting from programs of treadmill running and bicycle ergometer riding. Med Sci Sports 1972; 4: 6-10.

19 Gergley TJ et al. Specificity of arm training on aerobic power during swimming and running. Med Sci Sports Exerc 1984; 16: $349-354$. 